\title{
SCAPULAR METASTASIS BY RCC. CASE PRESENTATION
}

doi: $10.2478 /$ rojost-2018-0034

\author{
A.I. Nicula, A. Marinescu, A. Marinescu, G. Iana \\ "Carol Davila" University of Medicine and Pharmacy, Bucharest Romania \\ Department of Medical Imaging, University Emergency Hospital, Bucharest, Romania
}

Bony metastasis is a frequent occurrence in malignancies.

We present the case of a 75-year-old male patient who was investigated for a lytic lesion in the right scapula and was eventually diagnosed with metastatic renal cell carcinoma (RCC).

The main indications for embolization are reducing the risk of bleeding during and after surgery of hypervascular tumors, simplifying the manipulation of tumors, palliation of pain, bleeding, fever, and hypercalcemia-like symptoms in inoperable tumors, preventing further dissemination of a tumor, and increasing the response to chemotherapy and radiotherapy. Embolization may be a therapeutic alternative to surgery in cases in which surgery is inappropriate or associated with high risk.

In the case presented, CT examinations had a crucial role both in the diagnostic orientation and in the subsequent therapeutic decisions and proper monitoring under therapy.

Keywords: renal cell carcinoma, expansile bone metastasis 